\title{
Asymptotic Analysis of Asymmetric MIMO Links: EVM Limits for Joint Decoding of PSK and QAM
}

Mikko Vehkapera, Taneli Riihonen, Maksym Girnyk, Emil Björnson, Merouane Debbah, Lars K. Rasmussen and Risto Wichman

The self-archived postprint version of this conference article is available at Linköping University Institutional Repository (DiVA):

http:// urn.kb.se/ resolve?urn=urn:nbn:se:liu:diva- 127291

N.B.: When citing this work, cite the original publication.

Vehkapera, M., Riihonen, T., Girnyk, M., Björnson, E., Debbah, M., Rasmussen, L. K., Wichman, R., (2015), Asymptotic Analysis of Asymmetric MIMO Links: EVM Limits for J oint Decoding of PSK and QAM, 2015 IEEE INTERNATIONAL CONFERENCE ON COMMUNICATIONS (ICC) , 1869-1873. https:// doi.org/ 10.1109/ ICC.2015.7248597

Original publication available at:

https:// doi.org/ 10.1109/ ICC.2015.7248597

Copyright: IEEE

http://www.ieee.org/

(C)2015 IEEE. Personal use of this material is permitted. However, permission to reprint/republish this material for advertising or promotional purposes or for creating new collective works for resale or redistribution to servers or lists, or to reuse any copyrighted component of this work in other works must be obtained from the IEEE. 


\title{
Asymptotic Analysis of Asymmetric MIMO Links: EVM Limits for Joint Decoding of PSK and QAM
}

\author{
Mikko Vehkaperä ${ }^{1}$, Taneli Riihonen ${ }^{1}$, Maksym Girnyk ${ }^{2}$, Emil Björnson ${ }^{3}$, \\ Mérouane Debbah $^{4}$, Lars K. Rasmussen ${ }^{2}$, and Risto Wichman ${ }^{1}$ \\ ${ }^{1}$ Aalto University School of Electrical Engineering, Department of Signal Processing and Acoustics, Finland \\ ${ }^{2}$ KTH Royal Institute of Technology, ACCESS Linnaeus Centre and School of Electrical Engineering, Sweden \\ ${ }^{3}$ Linköping University, Department of Electrical Engineering (ISY), Sweden \\ ${ }^{4}$ Huawei France R\&D Center and Supélec, France \\ \{mikko.vehkapera, taneli.riihonen\}@aalto.fi, mgyr@kth.se, emil.bjornson@liu.se \\ merouane. debbah@huawei.com, lkra@kth.se, risto.wichman@aalto.fi
}

\begin{abstract}
Hardware non-idealities in wireless transmitter electronics cause distortion that is not captured by conventional linear channel models; in fact, error-vector magnitude (EVM) measurements in conformance testing conceptually reduce their collective effect to an additive noise component at each subcarrier. Motivated by the EVM, the present paper considers a 'binoisy' multiple-input multiple-output (MIMO) channel model where the additional non-idealities manifest themselves as an additive distortion noise term at the transmit side. Through this extended MIMO relation, the effects of hardware impairments on the achievable rates of different digital modulation schemes are studied via large system analysis. The numerical results illustrate how tolerable EVM levels depend non-trivially on various factors, including the signal-to-noise ratio, modulation order and the level of asymmetry in antenna array configurations.
\end{abstract}

\section{INTRODUCTION}

Digital, e.g., phase-shift keying (PSK) or quadrature amplitude modulation (QAM), data symbols are not discrete or deterministic due to hardware impairments [1], [2] after they pass a practical transmitter chain. Conformance testing measures this aspect through the error-vector magnitude (EVM) aiming at guaranteeing that communication is limited by either receiver noise or the lack of entropy in basic alphabets, e.g., QPSK. However, data rates have recently been improved by increasing modulation order, e.g., using 16-QAM at relatively high signal-to-noise ratio (SNR), in which cases transmitter hardware impairments can have significant impact and should be taken into account in theoretical performance analysis too.

In principle, the EVM characterizes the distortion effects as an additive transmitter noise term, which is in contrast with conventional link-level signal models that contain an additive thermal-noise term only at the receiver after a fading channel. Motivated by the EVM, we adopt the 'binoisy' channel model of [3]-[12], which includes also a transmit-side noise term; this is a convenient compromise between facilitating theoretical analysis and resorting to measurements or simulations. In a strict sense, additive noise is still only a simplified representation of complex nonlinear phenomena occurring due to hardware impairments, especially when considering their joint coupled effects or residual distortion after compensation.
The key reference results for the present study are reported in [3]-[12]. These seminal works formulated the research niche around the binoisy channel model and established the baseline understanding of MIMO communication under EVM with numerical simulations and theoretical analysis. However, all the earlier analytic capacity results are restricted to the notional case of Gaussian signaling while, except for [3], practical digital modulation schemes, e.g., PSK and QAM considered herein, have not been analytically evaluated for binoisy channels. On the other hand, simulation-based studies have mainly considered bit/symbol/packet error rates although the transmission rate is a more representative performance metric for modern systems which utilize adaptive encoding.

As for MIMO processing, we focus on regular spatial multiplexing, where a transmitter separately encodes and sends an independent data stream at each of its antennas without having channel state information or being aware of the transmit-side noise it produces, and a receiver jointly decodes the output signals of the fading MIMO channel knowing its instantaneous realization and the noise statistics. Mutual information (MI) between the channel input and output at the large-system limit (LSL) is evaluated for this scenario using the replica method ${ }^{1}$ [18], [19]. For some special cases like Gaussian signaling, we would have exact asymptotic results when the number of antennas grows without bound, while they can be otherwise considered accurate approximations for PSK and QAM as shown by comparisons to Monte Carlo simulations.

The analytic results, presented in a complete form in [20], allow us to study the rate loss due to EVM under joint decoding. The results cover all practical discrete modulation schemes for which QPSK, 8-PSK, and 16-QAM are used as examples in this paper. Especially, theoretical results are illustrated herein with a diverse set of new numerical results, including simulations for validating their accuracy. The focus is on understanding the effect of transmit-to-receive antenna ratio and finding the key worst-case scenarios which establishes practical guidelines for choosing target EVM limits.

\footnotetext{
${ }^{1}$ The replica method originates from statistical physics. It was introduced to the analysis of wireless systems by [13], [14] and later applied to various problems in communication theory, e.g., MIMO systems [15]-[17], by many.
} 


\section{SYSTEM MODEL}

Consider the system depicted in Fig. 1 and modeled as a binoisy channel [3]-[12]. The received signal is given by

$$
\boldsymbol{y}=\boldsymbol{H}(\boldsymbol{x}+\boldsymbol{v})+\boldsymbol{w},
$$

where $\boldsymbol{H} \in \mathbb{C}^{N \times M}$ is the MIMO channel matrix and $\boldsymbol{x} \in \mathbb{C}^{M}$ the signal of interest. The additive receive-side noise component $\boldsymbol{w} \in \mathbb{C}^{N}$ is caused by thermal noise and distortion arising from the non-ideal behavior of the radio-frequency (RF) transceivers. Similarly, $v \in \mathbb{C}^{M}$ represents the effect of thermal noise and hardware impairments at the transmit side. In conventional MIMO literature it is common to consider only the receive-side noise component, which translates to the special case of $\boldsymbol{v}=\mathbf{0}$ in our generic system model.

Let us denote the probability density (or mass) function of the transmit vector $\boldsymbol{x}$ by $p(\boldsymbol{x})$ and assume it factorizes as

$$
p(\boldsymbol{x})=\prod_{m=1}^{M} p\left(x_{m}\right),
$$

so that independent streams are transmitted at each transmit antenna. Furthermore, let $p\left(x_{m}\right)$ be a zero-mean distribution with variance $\bar{\gamma}_{m}$. For later convenience, we let $\boldsymbol{\Gamma}$ be a diagonal matrix whose non-zero elements are given by $\bar{\gamma}_{1}, \ldots, \bar{\gamma}_{M}$, that is, $\boldsymbol{\Gamma}=\mathrm{E}\left\{\boldsymbol{x} \boldsymbol{x}^{\mathrm{H}}\right\}$. The channel $\boldsymbol{H}$ is assumed to have independent identically distributed (IID) circularly symmetric complex Gaussian (CSCG) elements with variance ${ }^{2} 1 / M$. The additive noise vectors $\boldsymbol{v}$ and $\boldsymbol{w}$ are modeled as CSCG random vectors with covariance matrices $\boldsymbol{R}_{\boldsymbol{v}}$ and $\boldsymbol{R}_{\boldsymbol{w}}$, respectively. Since the hardware components causing distortions at each transmit-side branch are decoupled, we assume that the spatial covariance matrix of the noise term $\boldsymbol{v}$ is diagonal $\boldsymbol{R}_{\boldsymbol{v}}=$ $\operatorname{diag}\left(r_{\boldsymbol{v}}^{(1)}, \ldots, r_{\boldsymbol{v}}^{(M)}\right)$. Hence, $\boldsymbol{v}$ has independent entries drawn according to $p\left(v_{m}\right)=g\left(v_{m} \mid 0 ; r_{\boldsymbol{v}}^{(m)}\right)$. For simplicity we also assume that any given noise component is independent of any other random variable (RV) in the system. The SNR without transmit-side noise is defined as $\operatorname{tr}(\boldsymbol{\Gamma}) / \operatorname{tr}\left(\boldsymbol{R}_{\boldsymbol{w}}\right)$.

The probability density function (PDF) of the received signal, conditioned on $\boldsymbol{x}, \boldsymbol{v}$ and $\boldsymbol{H}$, is given by

$$
p(\boldsymbol{y} \mid \boldsymbol{x}, \boldsymbol{v}, \boldsymbol{H})=g\left(\boldsymbol{y} \mid \boldsymbol{H}(\boldsymbol{x}+\boldsymbol{v}) ; \boldsymbol{R}_{\boldsymbol{w}}\right),
$$

where $g(\boldsymbol{x} \mid \boldsymbol{\mu} ; \boldsymbol{R})$ denotes complex Gaussian density with mean $\boldsymbol{\mu}$ and covariance $\boldsymbol{R}$. The receiver is assumed to know $\boldsymbol{H}$ and the true distribution $p(\boldsymbol{x})$ of the channel input. However, the additional transmit-side term $\boldsymbol{v}$ is in general unknown at the receive side and, thus, the PDF in (3) cannot be directly used for detection and decoding. Herein, we consider the case where the receiver knows the PDFs of the noise plus distortion

\footnotetext{
${ }^{2}$ The assumption here is that the total power emitted from the transmit antennas is constant; that is, $\operatorname{tr}(\boldsymbol{\Gamma})=\bar{\gamma}$, where $\bar{\gamma}$ is some fixed power budget that does not depend on $M$. For the following analysis, however, it is more convenient to treat the elements of $\boldsymbol{\Gamma}$ to be independent of $M$ and let the transmit power normalization be part of the channel instead. We also use the shorthand $p(\boldsymbol{x})=g(\boldsymbol{x} \mid \boldsymbol{\mu} ; \boldsymbol{R})$ when $\boldsymbol{x}$ is a complex Gaussian RV with mean $\boldsymbol{\mu}$ and covariance matrix $\boldsymbol{R}$. Note that if RV $\boldsymbol{x}$ is CSCG, it has automatically zero mean so that defining the covariance matrix is sufficient for defining the density $p(\boldsymbol{x})$.
}

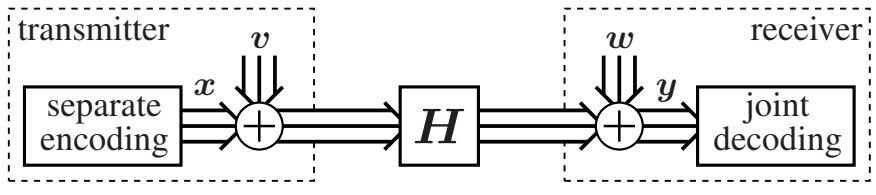

Fig. 1. System model for non-ideal MIMO communications with transmit and receive distortion. The receiver has perfect knowledge of the MIMO channel and statistical information about the additive distortion plus noise terms. Optimal joint decoding is performed at the receiver.

terms $\boldsymbol{v}$ and $\boldsymbol{w}$ as well as the distribution of the data vector $\boldsymbol{x}$ so that the conditional PDF

$$
\begin{aligned}
p(\boldsymbol{y} \mid \boldsymbol{x}, \boldsymbol{H}) & =\mathrm{E}_{\boldsymbol{v}}\left\{g\left(\boldsymbol{y} \mid \boldsymbol{H}(\boldsymbol{x}+\boldsymbol{v}) ; \boldsymbol{R}_{\boldsymbol{w}}\right)\right\} \\
& =g\left(\boldsymbol{y} \mid \boldsymbol{H} \boldsymbol{x} ; \boldsymbol{R}_{\boldsymbol{w}}+\boldsymbol{H} \boldsymbol{R}_{\boldsymbol{v}} \boldsymbol{H}\right)
\end{aligned}
$$

is used for joint decoding of the transmitted signals. Note that the effective noise covariance matrix in (5) depends now on the instantaneous channel realization $\boldsymbol{H}$.

\section{Performance AnAlysis}

Given that the input distribution at the transmitter is $p(\boldsymbol{x})$ and joint decoding with the channel transition probability (5) is performed at the receiver, the achievable rate of the system is described by the (ergodic) mutual information (MI) between the channel inputs and outputs. Herein we focus on the ideal case where codewords span infinitely many independent channel realizations and consider the ergodic MI:

$$
I(\boldsymbol{y} ; \boldsymbol{x})=\mathrm{E}\left\{\ln \left(\frac{p(\boldsymbol{y} \mid \boldsymbol{x}, \boldsymbol{H})\}}{\mathrm{E}_{\boldsymbol{x}}\{p(\boldsymbol{y} \mid \boldsymbol{x}, \boldsymbol{H})\}}\right)\right\},
$$

where the expectation is w.r.t. all RVs in (1). One of the entropy terms in (6), related to the numerator, reads

$$
\mathrm{E}\{\ln p(\boldsymbol{y} \mid \boldsymbol{x}, \boldsymbol{H})\}=-\mathrm{E}_{\boldsymbol{H}}\left\{\ln \operatorname{det}\left(\boldsymbol{R}_{\boldsymbol{w}}+\boldsymbol{H} \boldsymbol{R}_{\boldsymbol{v}} \boldsymbol{H}^{\mathrm{H}}\right)\right\}-c,
$$

where $c=N \ln (\mathrm{e} \pi)$ and we emphasized the fact that there is an expectation left w.r.t. the channel realizations $\boldsymbol{H}$ in (7). This could be evaluated, for example, using Monte Carlo methods or random matrix theory [21], [22]. Unfortunately, to the best of our knowledge, the other entropy term in (6), arising from the denominator, is mathematically intractable for rigorous methods like random matrix theory when $p(\boldsymbol{x})$ is an arbitrary distribution that satisfies (2). For example, given that the channel input for each transmit antenna is drawn uniformly at random from a finite set $\mathcal{A}$, calculating $\mathrm{E}\{\ln p(\boldsymbol{y} \mid \boldsymbol{H})\}$ and combining it with (7) reduces (6) to the form (8) given at the top of the next page. This form is computationally very complex and can be evaluated using Monte Carlo methods only for small number of antennas and simple constellations.

We use the replica method to obtain analytic results for a general input distribution $p(\boldsymbol{x})$. They are written in a compressed form where the assumption of LSL is suppressed for notational simplicity. For finite $M$ and $N$, the results should therefore be considered as approximations. The derivations have been omitted here due to space constraints but they can be found, among other complementing results, in the related journal article [20] and the current numerical results are new. 


$$
I(\boldsymbol{y} ; \boldsymbol{x})=M \ln |\mathcal{A}|-N-\frac{1}{|\mathcal{A}|} \sum_{\boldsymbol{x} \in \mathcal{A}^{M}} \mathrm{E}_{\boldsymbol{v}, \boldsymbol{w}, \boldsymbol{H}}\left\{\ln \left(\sum_{\tilde{\boldsymbol{x}} \in \mathcal{A}^{M}} \mathrm{e}^{-[\boldsymbol{H}(\boldsymbol{x}-\tilde{\boldsymbol{x}}+\boldsymbol{v})+\boldsymbol{w}]^{\mathrm{H}}\left(\boldsymbol{R}_{\boldsymbol{w}}+\boldsymbol{H} \boldsymbol{R}_{v} \boldsymbol{H}\right)^{-1}[\boldsymbol{H}(\boldsymbol{x}-\tilde{\boldsymbol{x}}+\boldsymbol{v})+\boldsymbol{w}]}\right)\right\}
$$

Proposition 1. Let us define for notational convenience a scalar channel input

$$
\chi_{m}=x_{m}+v_{m},
$$

where $x_{m} \sim p\left(x_{m}\right)$ and $v_{m} \sim g\left(v_{m} \mid 0 ; r_{\boldsymbol{v}}^{(m)}\right)$ are independent for all $m=1, \ldots, M$. Furthermore, let

$$
p\left(z_{m} \mid \chi_{m}\right)=g\left(z_{m} \mid \chi_{m} ; \eta^{-1}\right)
$$

be a conditional PDF of a non-fading single-input singleoutput (SISO) AWGN channel whose input is (9) and the noise variance is given by $\eta^{-1}$. The conditional mean estimator of $\chi_{m}$ received over this channel reads then by definition

$$
\left\langle\chi_{m}\right\rangle=\frac{\mathrm{E}_{\chi_{m}}\left\{\chi_{m} p\left(z_{m} \mid \chi_{m}\right)\right\}}{\mathrm{E}_{\chi_{m}}\left\{p\left(z_{m} \mid \chi_{m}\right)\right\}}
$$

where the parameter $\eta$ is given, along with another parameter $\varepsilon$, as the solution to the coupled fixed point equations

$$
\begin{aligned}
& \eta=\frac{1}{\alpha N} \operatorname{tr}\left[\left(\boldsymbol{R}_{\boldsymbol{w}}+\varepsilon \boldsymbol{I}_{N}\right)^{-1}\right], \\
& \varepsilon=\frac{1}{M} \sum_{m=1}^{M}\left[\bar{\gamma}_{m}+r_{\boldsymbol{v}}^{(m)}-\mathrm{E}\left|\left\langle\chi_{m}\right\rangle\right|^{2}\right] .
\end{aligned}
$$

If we also define a second set of parameters $\eta^{\prime}$ and $\varepsilon^{\prime}$ that are solutions to another set of coupled fixed point equations, independent of (12) and (13),

$$
\begin{aligned}
& \eta^{\prime}=\frac{1}{\alpha N} \operatorname{tr}\left[\left(\boldsymbol{R}_{\boldsymbol{w}}+\varepsilon^{\prime} \boldsymbol{I}_{N}\right)^{-1}\right] \\
& \varepsilon^{\prime}=\frac{1}{M} \sum_{m=1}^{M} \frac{r_{\boldsymbol{v}}^{(m)}}{1+\eta^{\prime} r_{\boldsymbol{v}}^{(m)}}
\end{aligned}
$$

the per-stream ergodic MI of the original MIMO system (6) can be approximated as

$$
\begin{aligned}
& \frac{1}{M} I(\boldsymbol{y} ; \boldsymbol{x})=\frac{\ln \operatorname{det}\left(\boldsymbol{R}_{\boldsymbol{w}}+\varepsilon \boldsymbol{I}_{N}\right)-\ln \operatorname{det}\left(\boldsymbol{R}_{\boldsymbol{w}}+\varepsilon^{\prime} \boldsymbol{I}_{N}\right)}{\alpha N} \\
& -\left(\eta \varepsilon-\eta^{\prime} \varepsilon^{\prime}\right)+\frac{1}{M} \sum_{m=1}^{M}\left[I\left(z_{m} ; \chi_{m}\right)-\ln \left(1+\eta^{\prime} r_{\boldsymbol{v}}^{(m)}\right)\right],
\end{aligned}
$$

where $\alpha=M / N$ and $I\left(z_{m} ; \chi_{m}\right)$ is the MI of the Gaussian channel defined by (9) and (10).

Proposition 1 holds for all input distributions that satisfy (2), including notional Gaussian signals, and exemplifies the so-called decoupling principle [14]. Herein this means that the analysis of a fading MIMO channel can be decomposed to analyzing an equivalent set of non-fading SISO channels. For concreteness, we concentrate next on the case of discrete signaling when the noise plus distortion is spatially white $\left(\boldsymbol{R}_{\boldsymbol{v}}=r_{\boldsymbol{v}} \boldsymbol{I}_{M}\right)$ and the transmit power is uniformly allocated $\left(\boldsymbol{\Gamma}=\bar{\gamma} \boldsymbol{I}_{M}\right)$. This makes the channels $m=1,2, \ldots, M$ identically distributed and the subscript $m$ can be omitted.
Example 1. Let $\mathcal{A}$ be a discrete modulation alphabet with fixed and finite cardinality $|\mathcal{A}|$. Let the channel inputs $x_{m}$ be drawn independently and uniformly from $\mathcal{A}$. The parameter $\varepsilon$ in (13) is obtained using ${ }^{3}$

$$
\begin{gathered}
\langle\chi\rangle=\frac{1}{p(z)} \sum_{x \in \mathcal{A}}\left[\frac{1}{|\mathcal{A}|} g\left(z \mid x ; \eta^{-1}+r_{\boldsymbol{v}}\right)\left(\frac{x+\eta r_{\boldsymbol{v}} z}{1+\eta r_{\boldsymbol{v}}}\right)\right], \\
\mathrm{E}\left\{|\langle\chi\rangle|^{2}\right\}=\int_{\mathbb{C}} p(z) \mathrm{E}\left\{|\langle\chi\rangle|^{2}\right\} \mathrm{d} z,
\end{gathered}
$$

in Proposition 1. Here $p(z)$ is given by

$$
p(z)=\frac{1}{|\mathcal{A}|} \sum_{x \in \mathcal{A}} g\left(z \mid x ; \eta^{-1}+r_{\boldsymbol{v}}\right),
$$

and the related MI term reads by definition as

$$
I(z ; \chi)=\ln \left(\frac{\eta}{\mathrm{e} \pi}\right)-\int_{\mathbb{C}} p(z) \ln p(z) \mathrm{d} z .
$$

In order to obtain (16), both (13) and (20) need to be solved in general numerically.

\section{NUMERICAL EXAMPLES}

In the following, assume for simplicity that $\boldsymbol{\Gamma}=\bar{\gamma} \boldsymbol{I}, \boldsymbol{R}_{\boldsymbol{w}}=$ $\boldsymbol{I}$ and $\boldsymbol{R}_{\boldsymbol{v}}=\left(10^{\mathrm{EVM} / 10}\right) \bar{\gamma} \boldsymbol{I}$, where EVM gives the error-vector magnitude of the transmitter in decibels. The SNR without transmit-side noise is therefore simply $\bar{\gamma}$.

The first numerical experiment plotted in Fig. 2 examines the accuracy of the asymptotic analytic results when applied to finite-sized systems. The EVM is fixed to the rather pessimistic value of $E V M=-10 \mathrm{~dB}$ to highlight the differences between the ideal and imperfect hardware configurations. The normalized rate is shown using the asymptotic replica analysis (lines) and Monte Carlo simulations (markers) for a finitesize symmetric antenna setup with $M=N=4$. The gap between asymptotic result presented in Example 1 and Monte Carlo averaging of (8) is somewhat greater for the ideal case than for the noisy setup with hardware impairments. The analytic approximation given by the replica method is, however, reasonably good already at $M=N=4$, even though $M, N \rightarrow \infty$ is required formally by the analysis.

The next example in Fig. 3 illustrates the performance of a MIMO system with three different antenna ratios $\alpha=M / N \in$ $\{1 / 2,1,2\}$. To emphasize the effect of hardware impairments, we have plotted the rate loss (in percents) for the case with transmit noise $(E V M=-20 \mathrm{~dB}$ ) when compared to the ideal case. The markers depict the points where the maximum relative rate loss is experienced for joint decoding; except for the case of 16-QAM and $\alpha=2$ that has maximum EVM loss at $\bar{\gamma}=25.5 \mathrm{~dB}$ and hence outside of the range of the figure.

\footnotetext{
${ }^{3}$ For a complex variable $z=x+\mathrm{j} y$, we denote $\int_{\mathbb{C}}() \mathrm{d} z=\int_{\mathbb{R}^{2}}() \mathrm{d} x \mathrm{~d} y$.
} 


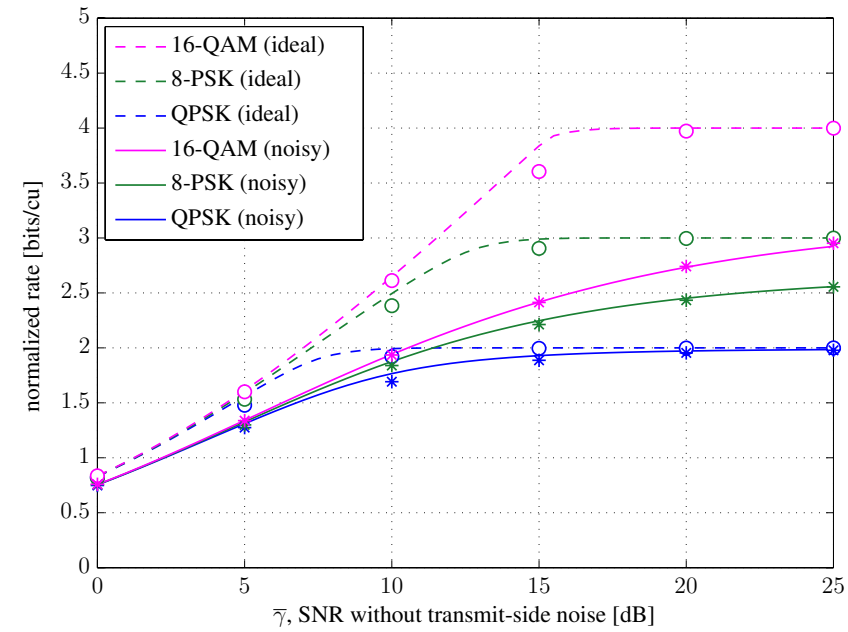

Fig. 2. Normalized rate $M^{-1} I(\boldsymbol{y} ; \boldsymbol{x})$ in bits per channel use (cu) vs. SNR for MIMO transmission with ideal hardware (EVM $=-\infty \mathrm{dB}$ ) and hardware impairments $(E V M=-10 \mathrm{~dB})$. Lines are for replica results and markers for Monte Carlo simulations with $M=N=4$ antenna configuration.

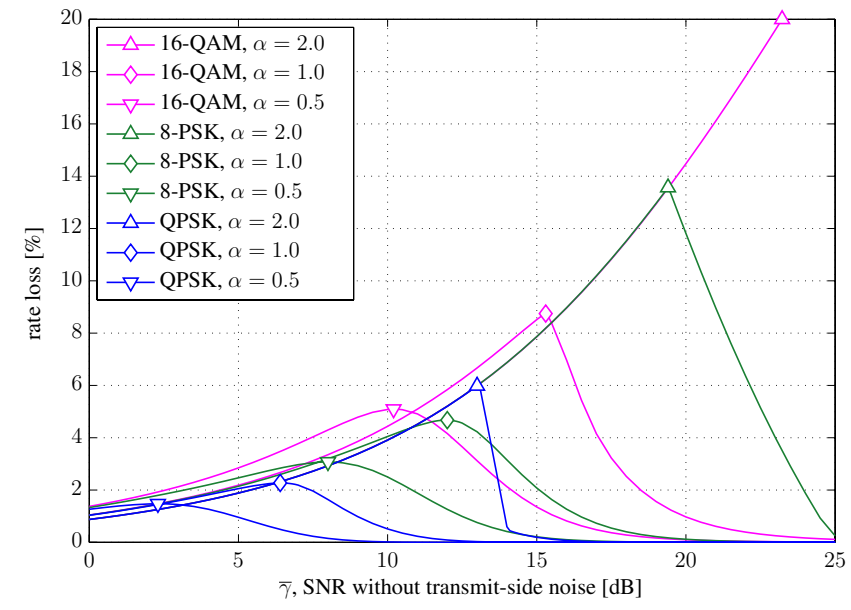

Fig. 3. Rate loss percentage vs. SNR for noisy setup (EVM $=-20 \mathrm{~dB})$ when compared to ideal hardware. Three different antenna ratios, $\alpha=$ $M / N \in\{1 / 2,1,2\}$, are shown. The markers depict the maximum rate loss (in percentage) for each of the cases within the given SNR region.

Adopting the worst-case SNRs revealed by Fig. 3 as representative example values, Fig. 4 illustrates the rate loss due to transmit-side noise in terms of the ratio of the number of transmit antennas to the number of receive antennas. In principle, having a large number of receive antennas compared to transmit antennas yields SNR gain which effectively compensates for the existence of transmit-side noise. When increasing the antenna ratio, the rate loss increases until it reaches a worstcase scenario, which depends on the SNR operation point, after which it decreases slightly until converging to a saturation level. The transmit-side noise is especially harmful when a large number of spatial streams are transmitted in comparison to the number of receive antennas. Interestingly, the worst-case scenarios are not exactly the same when they are determined based on SNR (cf. Fig. 3) and the antenna ratio (cf. Fig. 4).

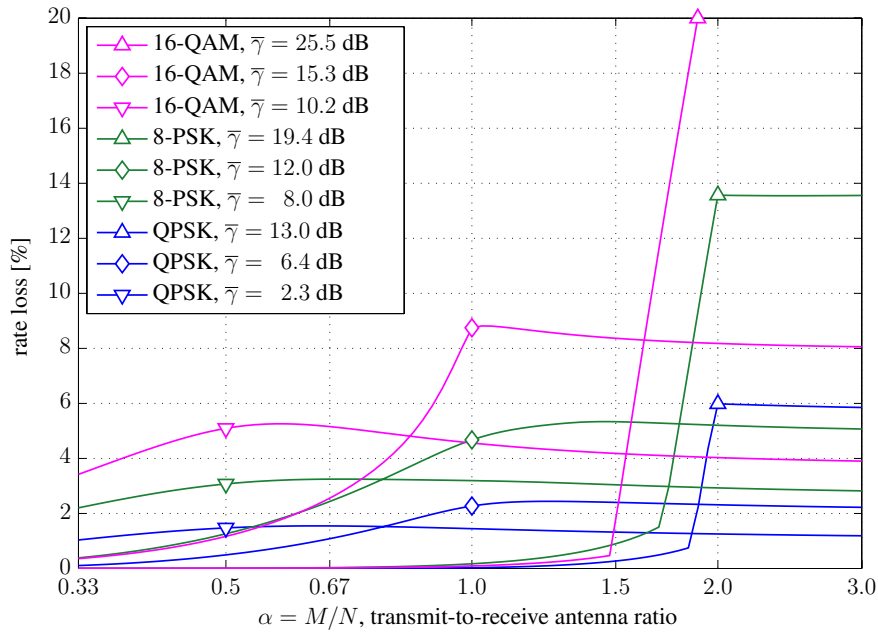

Fig. 4. Rate loss percentage vs. antenna ratios $\alpha=M / N$ for noisy setup $(E V M=-20 \mathrm{~dB})$ when compared to ideal hardware at different SNRs. The SNRs are selected to match the markers found in Fig. 3, except for the high-SNR case of 16-QAM that corresponds to maximum rate loss at $\alpha=2$.

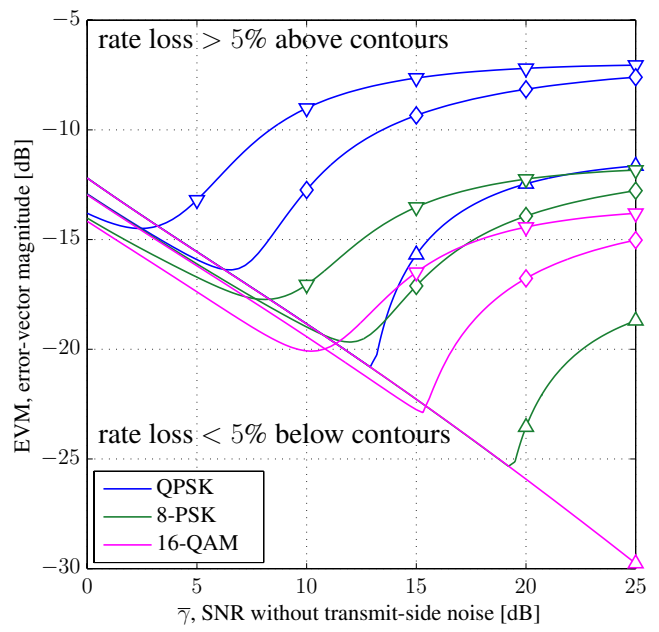

Fig. 5. The $5 \%$ rateloss contours for three different antenna ratios $\alpha=$ $M / N \in\{1 / 2,1,2\}$ as a function of SNR and EVM. The regions above the curves define the $(\bar{\gamma}$, EVM) pairs that result to rate losses higher than $5 \%$, and vice versa for the areas below the curves. For the full legend, see Fig. 3.

Finally, Fig. 5 represents the most processed form of the new numerical results generated based on the replica analysis to illustrate the ultimate EVM limits in efficient operation. One should note that it would be nearly impossible, or at least computationally prohibitive, to obtain equivalent results from simulations unless considering Gaussian signaling, which demonstrates the value of our theoretical study for the analysis of digital modulation. In particular, Fig. 5 shows contours for a rate loss of $5 \%$ in terms of both SNR and EVM such that the MIMO link should operate below the curves to meet this specific, reasonably tolerable, target when its transmit-to-receive antenna ratio is fixed at the example values $\alpha=M / N \in\{1 / 2,1,2\}$. The lowest points of the curves yield the ultimate worst-case scenarios in terms of SNR and EVM limits below which the rate loss is small at any SNR. 
TABLE I

SUGGESTED EVM TARGETS FOR DIGITAL MODULATION

\begin{tabular}{|c|c|c|c|}
\hline \multicolumn{4}{|c|}{ transmit-to-receive antenna ratio, $\alpha=M / N$} \\
\hline constellation & $1 / 2$ & 1 & 2 \\
\hline QPSK & $-15 \mathrm{~dB}$ & $-17 \mathrm{~dB}$ & $-21 \mathrm{~dB}$ \\
\hline 8-PSK & $-18 \mathrm{~dB}$ & $-20 \mathrm{~dB}$ & $-26 \mathrm{~dB}$ \\
\hline 16-QAM & $-21 \mathrm{~dB}$ & $-23 \mathrm{~dB}$ & $-31 \mathrm{~dB}$ \\
\hline 64-QAM* & & $-29 \mathrm{~dB}$ & \\
\hline
\end{tabular}

${ }^{*}$ The target for 64-QAM with symmetric antenna configuration is concluded from the numerical results of [20] for comparison.

\section{Discussion AND CONCLUSIONS}

Considering a 'binoisy' channel model, we studied the asymptotic (the number of transmit $M$ and receive $N$ antennas grow without bound while their ratio $\alpha$ is fixed) achievable rates of spatially multiplexed MIMO systems suffering from transceiver hardware impairments. For optimal joint decoding, where the receiver is designed and implemented explicitly based on the generalized system model, expressions have been given for the ergodic mutual information between the channel inputs and outputs. The mathematical expressions provided in the paper cover practical discrete modulation schemes such as QAM and PSK, as well as capacity-achieving Gaussian signaling. Finite-sized simulations were provided to verify the accuracy of the asymptotic expressions.

The numerical results showed that the effects of transmitside noise depend non-trivially on multiple factors, including the signal-to-noise ratio, modulation order and the level of asymmetry in antenna array configurations. The results were also used to identify the EVM values that allow for system operation within a certain maximum rate loss percentage compared to the ideal case. In the considered cases, the asymmetry in antenna array configurations had the effect of either increasing the relative rate loss $(M>N)$ or decreasing the effect of transmit-side distortion $(M<N)$ when compared to the standard, symmetric setup $M=N$, for the same modulation order, EVM and SNR. Especially, the rate loss is pronounced when the modulation order is increased and there are a large number of independent transmit streams.

As an ultimate conclusion, we suggest a set of EVM targets deduced from the numerical results to provide guidelines for practical conformance testing and for justifying the cases where conventional theoretical studies can convincingly neglect transmitter hardware impairments: If the EVM remains below the values tabulated in Table I, their effect can be considered negligible. Rate loss is usually much less than $5 \%$ if the SNR differs from a worst-case scenario and the antenna ratio is less than the tabulated value. The values have also been rounded down to $1 \mathrm{~dB}$ precision which adds safety margin.

\section{ACKNOWLEDGMENT}

This work was partially supported by the Academy of Finland, the Swedish Research Council (VR) and the ERC Starting Grant 305123 MORE.

\section{REFERENCES}

[1] G. Fettweis, M. Löhning, D. Petrovic, M. Windisch, P. Zillmann, and W. Rave, "Dirty RF: A new paradigm," International Journal of Wireless Information Networks, vol. 14, no. 2, pp. 133-148, June 2007.

[2] T. Schenk, RF Imperfections in High-rate Wireless Systems: Impact and Digital Compensation. Springer, 2008.

[3] T. C. W. Schenk, P. F. M. Smulders, and E. R. Fledderus, "Performance of MIMO OFDM systems in fading channels with additive TX and RX impairments," in Proc. 1st Annual IEEE BENELUX/DSP Valley Signal Processing Symposium, April 2005.

[4] B. Göransson, S. Grant, E. Larsson, and Z. Feng, "Effect of transmitter and receiver impairments on the performance of MIMO in HSDPA," in Proc. 9th IEEE Workshop on Signal Processing Advances in Wireless Communications, July 2008.

[5] H. Suzuki, T. V. Anh Tran, I. B. Collings, G. Daniels, and M. Hedley, "Transmitter noise effect on the performance of a MIMO-OFDM hardware implementation achieving improved coverage," IEEE Journal on Selected Areas in Communications, vol. 26, no. 6, pp. 867-876, August 2008.

[6] H. Suzuki, I. B. Collings, M. Hedley, and G. Daniels, "Practical performance of MIMO-OFDM-LDPC with low complexity double iterative receiver," in Proc. 20th IEEE International Symposium on Personal, Indoor and Mobile Radio Communications, September 2009.

[7] C. Studer, M. Wenk, and A. Burg, "MIMO transmission with residual transmit-RF impairments," in Proc. International ITG Workshop on Smart Antennas, February 2010.

[8] J. González-Coma, P. M. Castro, and L. Castedo, "Impact of transmit impairments on multiuser MIMO non-linear transceivers," in Proc. International ITG Workshop on Smart Antennas, February 2011.

[9] C. Studer, M. Wenk, and A. Burg, "System-level implications of residual transmit-RF impairments in MIMO systems," in Proc. 5th European Conference on Antennas and Propagation, April 2011.

[10] J. González-Coma, P. M. Castro, and L. Castedo, "Transmit impairments influence on the performance of MIMO receivers and precoders," in Proc. 11th European Wireless Conference, April 2011.

[11] E. Björnson, P. Zetterberg, M. Bengtsson, and B. Ottersten, "Capacity limits and multiplexing gains of MIMO channels with transceiver impairments," IEEE Communications Letters, vol. 17, no. 1, pp. 9194, January 2013.

[12] X. Zhang, M. Matthaiou, E. Björnson, M. Coldrey, and M. Debbah, "On the MIMO capacity with residual transceiver hardware impairments," in Proc. IEEE International Conference on Communications, June 2014.

[13] T. Tanaka, "A statistical-mechanics approach to large-system analysis of CDMA multiuser detectors," IEEE Transactions on Information Theory, vol. 48, no. 11, pp. 2888-2910, November 2002.

[14] D. Guo and S. Verdú, "Randomly spread CDMA: Asymptotics via statistical physics," IEEE Transactions on Information Theory, vol. 51, no. 6, pp. 1983-2010, June 2005.

[15] R. R. Müller, "Channel capacity and minimum probability of error in large dual antenna array systems with binary modulation," IEEE Transactions on Signal Processing, vol. 51, no. 11, pp. 2821-2828, November 2003.

[16] C.-K. Wen, K.-K. Wong, and J.-C. Chen, "Spatially correlated MIMO multiple-access systems with macrodiversity: Asymptotic analysis via statistical physics," IEEE Transactions on Communications, vol. 55, no. 3, pp. 477-488, March 2007.

[17] K. Takeuchi, R. R. Müller, M. Vehkaperä, and T. Tanaka, "On an achievable rate of large rayleigh block-fading MIMO channels with no CSI," IEEE Transactions on Information Theory, vol. 59, no. 10, pp. 6517-6541, October 2013.

[18] H. Nishimori, Statistical Physics of Spin Glasses and Information Processing. New York: Oxford University Press, 2001.

[19] M. Mézard and A. Montanari, Information, Physics, and Computation. New York: Oxford University Press, 2009.

[20] M. Vehkaperä, T. Riihonen, M. A. Girnyk, E. Björnson, M. Debbah, L. K. Rasmussen, and R. Wichman, "Asymptotic analysis of SU-MIMO channels with transmitter noise and mismatched joint decoding," IEEE Transactions on Communications, 2015 (arXiv:1406.4980 [cs.IT]).

[21] A. M. Tulino and S. Verdú, "Random matrix theory and wireless communications," Foundations and Trends in Communications and Information Theory, vol. 1, no. 1, pp. 1-182, 2004.

[22] R. Couillet and M. Debbah, Random Matrix Methods for Wireless Communications. Cambridge University Press, 2011. 